A. Pramesh Rao, G. Swarup and Gopal-Krishna, eds.

\title{
Coma Southwest - as seen by the GMRT
}

\author{
K. S. Dwarakanath \\ Raman Research Institute, Bangalore 560080, India
}

\author{
J.N. Chengalur \\ National Center for Radio Astrophysics, TIFR, Post Bag No 3, \\ Ganeshkhind, Pune 41007, India
}

\begin{abstract}
We have imaged in $\mathrm{HI}$ a $\sim 25^{\prime}$ field of view in the Coma Southwest region using the Giant Meterwave Radio Telescope. No HI was detected to a $3 \sigma$ limit of $3 \times 10^{8} M_{\odot}$ in the surveyed volume. Except for NGC 4839, no radio continuum emission was detected from the galaxies in the Coma Southwest to a $3 \sigma$ limit of $3 \times 10^{20} \mathrm{~W} \mathrm{~Hz}^{-1}$ of spectral luminosity at $1.4 \mathrm{GHz}$.
\end{abstract}

\section{Introduction}

The Coma cluster of galaxies is a nearby cluster at a systemic velocity of $\sim 7000 \mathrm{~km} \mathrm{~s}^{-1}$. A ROSAT X-ray image of this region shows a clump of emission to the southwest of the main cluster emission (Caldwell et al 1993). This southwest region is also interesting in having many abnormal spectrum galaxies. The motivations for the present exercise are three fold : (a) to use the GMRT as a spectral line imaging instrument and compare its performance with that expected, (b) to image a nearby cluster in $\mathrm{HI}$ and radio continuum as a first step towards imaging higher redshift clusters $(\mathrm{z}=0$ to 0.5$)$ using the wide-band capabilities of the $21 \mathrm{~cm}$ feeds of GMRT, and (c) detect radio continuum and/or HI from galaxies in the Coma Southwest region.

\section{Observations and Preliminary Results}

The recently completed Giant Meterwave Radio Telescope (GMRT) was used for the observations reported here. Although the GMRT has 30 antennas spread over $\sim 25 \mathrm{~km}$, only the 10 antennas in the central $1 \mathrm{~km}^{2}$ area were used during the current observations. The antennas were pointed at $\alpha(\mathrm{J} 2000)=12^{h} 57^{m}$, and $\delta(\mathrm{J} 2000)=27^{0} 30^{\prime}$. The full width at half maximum of the primary beam of the GMRT dishes is $\sim 25^{\prime}$. Two velocity settings were used to cover the range from $4500 \mathrm{~km} \mathrm{~s}^{-1}$ to $9500 \mathrm{~km} \mathrm{~s}^{-1}$ with a velocity resolution of $\sim 26 \mathrm{~km} \mathrm{~s}^{-1}$. A total observing session of $2 \times 8$ hours was carried out. Continuum images as well as continuum subtracted spectral cubes of the observed region were produced. The images were smoothed to a synthesized beam of $30^{\prime \prime}$ (full width at half 
maximum). The rms achieved in the line image was $\sim 0.85 \mathrm{mJy} / \mathrm{beam} / \mathrm{channel}$, and $\sim 0.1 \mathrm{mJy} /$ beam in the continuum image.

The sensitivities achieved are consistent with that expected after allowing for about $30 \%$ of corrupted data. No systematics were detected either in the continuum image or in the spectral cube at the current sensitivities. No HI was detected to a $3 \sigma$ limit of $3 \times 10^{8} \mathrm{M}_{\odot}$ in the surveyed volume of $\sim 20 \mathrm{Mpc}^{3}$ $\left(\mathrm{H}_{o}=75 \mathrm{~km} \mathrm{~s}^{-1} \mathrm{Mpc}^{-1}\right.$, line width $\left.=100 \mathrm{~km} \mathrm{~s}^{-1}\right)$. In the radio continuum image only NGC 4839 was detected at a peak flux density of $18 \mathrm{mJy} /$ beam. A comparison of the radio sources and all galaxies from the NASA Extragalactic Database within the surveyed volume showed that none of these galaxies were detected to a $3 \sigma$ limit of $3 \times 10^{20} \mathrm{~W} \mathrm{~Hz}^{-1}$ of spectral luminosity at $1.4 \mathrm{GHz}$.

\section{Discussion}

The HI mass limit reached in the current observations is sensitive enough to detect even anemic galaxies. For e.g., in the Virgo cluster even the anemic galaxies have $\mathrm{HI}$ masses greater than $10^{8} \mathrm{M}_{\odot}$. In the Abell 2670 cluster $\left(\mathrm{V}_{\text {sys }} \sim\right.$ $23000 \mathrm{~km} \mathrm{~s}^{-1}$ ) which has been imaged in HI with the VLA, $\sim 10$ galaxies with HI masses greater than the current limit were detected in the southwest pointing. However, the volume surveyed in the southwest pointing in Abell 2670 was $370 \mathrm{Mpc}^{3}$. In the current observations, despite the large velocity coverage, the volume surveyed in a single pointing is $\sim 20 \mathrm{Mpc}^{3}$ due to the nearness of the Coma cluster. From this point of view it is premature to draw any conclusions at the moment about the gas content of galaxies in the Coma SW. However, it is interesting to speculate. There are arguments to indicate that Coma SW has already passed through the Coma cluster and might be turning around (Burns et al 1994), or, it is falling in for the first time (Colless \& Dunn 1996). In the former case, we might not expect to detect much $\mathrm{HI}$ from the galaxies in Coma $\mathrm{SW}$, while if the latter were correct we might expect to detect a few galaxies in $\mathrm{HI}$ if the survey is extended to a larger volume at a lower (a few times) detection limit.

Another interesting aspect of Coma SW is its large fraction of galaxies which exhibit enhanced Balmer absorption lines or emission lines, indicative of recent star formation or nuclear activity. There are also galaxies with abnormal spectra with [OII] emission lines too weak for the enhanced Balmer absorption. The absence of radio continuum from any of these galaxies (except for NGC 4839) indicates that these galaxies are quite 'normal' in this respect. A deeper continuum image is required to detect normal radio emission from these galaxies.

\section{References}

Caldwell, N., Rose, J. A., Sharples, R. M., Ellis, R. S., \& Bower, R. G. 1993, AJ, 106, 473.

Burns, J.O., Roettiger, K., Ledlow, M., \& Klypin, A. 1994, ApJ, 427, L87.

Colless, M., \& Dunn, A. M. 1996, ApJ, 458, 435. 